\title{
Is Time an Abstract Entity?
}

\author{
Jan Faye, Copenhagen
}

\section{Introduction}

Time is a shadowy entity. On the one hand, it appears elusive, intangible, and invisible. What we see is not time itself but changes of things and thoughts. In its proper form time seems to be given to us in the immediate experience as things and thoughts undergoing change prior to any conceptual understanding. Thus immediate experience and the temporal consciousness of the world have profound implications for how we reach a grasp of time. Some philosophers therefore think that the actuality of the present is a fundamental property of any change and see this feature as a manifestation of temporal becoming from the past to the future. On the other hand, modern physics takes the combination of space and time to be a real entity which may even have an influence on the distribution of energy and matter. This is why others maintain that the study of physics can give us a complete and correct answer concerning the nature of time.

It is in this perspective that I shall suggest that time is not a concrete but an abstract particular. If we apply all the common criteria of abstractness, it turns out that time is able to meet each and every one of them. I think this is because our concept of time is a result of an abstraction from our experience of concrete, physical things undergoing change. We need such a concept in order to be able to name and measure such things and events. But this does not make time physically real. As an abstract entity time consists of the set of all changes in the world.

\section{Kant on time}

In his Kritik der reinen Vernunft Kant argued that space and time are forms of intuition. What he meant by that was that space and time are necessary representations that underlie all perceptions. We cannot, he said, remove space and time with respect to the phenomena in general even though we may quite well think of space and time devoid of any phenomena. Space and time are given a priori as universal preconditions for the existence of 
phenomena, and therefore cannot vanish, whereas the phenomena may all vanish. In addition, Kant maintained that space and time are in us. This should not be taken literally. 'In us' in the transcendental sense means only that space and time are not things in themselves but are mind-dependent. As forms of intuitions they are not concepts because concepts have instances, like the concept of red which has red things as its instances. In contrast, forms of space and time do not have instances since a part of space and a moment of time are merely a part and a moment and not an instance of space and time. Thus, Kant argued explicitly with respect to time: 1) it is not an empirical concept; 2) it cannot be excluded from thought but it is a necessary representation; 3 ) it is not a discursive concept but an a priori form of intuition; and finally 4) it is an infinite entity which consists of an infinite number of moments or segments of times because time as something unlimited is a necessary precondition for speaking about what is limited in time.

Kant also discussed what he called the antinomies of the pure reason. The first of these antinomies was the thesis that the world has a beginning in time and has a limited extension in space in contrast to the antithesis according to which the world has no beginning and no boundaries. His intention was to show that the thesis and the antithesis both seem valid and that they could be proved on necessary a priori grounds. The proof of the thesis attempts to show that the opposite does not hold. Assume the world had no beginning. This implies that there are no moments back in time where it would not be correct to say that the world had already existed for infinity. Therefore the world up to now must have developed through an infinite series of events. This means that we have an infinite series which must be completed. But an infinite series can never be finished. Hence the world must have a beginning in Time. The proof rests on the assumption that we can apply the principle of pure reason to phenomena, namely that if the conditioned is given, the totality of the conditions is given, and therefore the unconditioned must be given.

Also the proof of the antithesis attempts to show that the opposite does not hold: if the world began in time, then nothing existed before the world began. But from nothing something cannot arise. In empty time no becoming and no beginning is possible. Therefore the world must be infinite in time. This proof presupposes that the world of phenomena is the world of things-in-themselves. It treats time as if it is an objective reality in itself. 
Now, both the thesis and the antithesis cannot be true. The solution, according to Kant, is to realize that when we talk about the thesis we consider the world to be a thing-in-itself, and when we talk about the antithesis we think of the world as it appears to us in time and space. Since every beginning takes place in space and time, the world-in-itself cannot begin in space and time, and since every phenomenon is conditioned by another phenomenon, the world as a phenomenon cannot be limited by empty space and empty time which cannot be objects of perception. The world, said Kant, is neither finite nor infinite because the series of possible events does neither have a first nor a final element, and the series of actual events could always be extended in infinitum.

Modern cosmology has a very different story to tell. This story has it that not only did the universe begin in a Big Bang around 14 billions years ago, where it was squeezed into a tiny little thing, but space and time themselves came into existence when the Big Bang took place. There were no instants earlier than the Big Bang and therefore no instance before where the Big Bang had yet not occurred. Ever since, the universe has expanded, not in space, but by adding more and more space between the galaxies. The universe consists of expanding space. As the universe expands, it moves forward towards the future, and the claim is that the direction of time can be explained in terms of the arrow of the expansion or in terms of the arrow of thermodynamics which then can be associated with this expansion. The motivation for saying so seems partly to stem from the close connection between the distribution of matter and energy and the curvature of space-time that we encounter in Einstein's field equations.

The important difference between Kant's view and the view of modern cosmology is this: Kant denied that space and time are things-in-themselves, which are exactly what modern cosmology assumes that they are. Although space and time, for Kant, are not concepts, but forms of intuition, they behave, like concepts, as abstract entities. This interpretation could indeed be challenged. Because Kant also argued that space and time are the subjective conditions of the possibility of perception. What he meant by calling them subjective was apparently not that their existence depends on the existence of a sensory being. Rather he believed that space and time are subjective in the sense that they are a priori forms of intuition and therefore preconditions for our experience of the empirical reality. As a priori forms of intuition, space and time may still be abstract entities, but as preconditions they could not exist as abstractions from experience. 


\section{Time as an abstract entity}

Something can, indeed, be abstract by failing to meet the criteria for concreteness, and yet be entirely mind-independent (e.g. numbers, as the Platonist conceives of them). So an argument for the abstract nature of time is not a vindication of Kant's position. Thus, regardless of the actual ontological status of space and time in Kant's philosophy, what can be said about phenomena is not the same as what can be said about space and time. For instance, we can, and must, say that physical phenomena are in space and time, but we cannot say that space and time are in space and time. Accordingly, it is an empirical fact, Kant would say, that all phenomena must begin at a time. The universe as an empirically studied object may therefore have an age. This does not, however, allow us to say analogically that time must begin at a time. Quite the opposite, behaving like an abstract entity time cannot have a beginning and therefore cannot have an age.

Modern cosmology follows Newton and Leibniz by assuming that space and time are concrete particulars. Newton believed not only that space and time contain an absolute spatial and temporal metric, but also consist of real spatial points and real temporal moments, existing independently of the events that may or may not take place at them. Where Newton thought that both are absolute by having a nature independent of material objects, and therefore could exist before the world was created, Leibniz believed that they are reducible to spatial and temporal relations between physical things and their changes and movements. Thus, Leibniz did reject, in his correspondence with Clarke, that space and time could exist before the world was created. Their differences apart, the similarity between Newton and Leibniz is exactly that they treated space and time as something concrete that could be studied empirically. Newton thought that absolute motion could be considered evidence for absolute space and time which in themselves were intangible and invisible. Leibniz, on the other hand, saw the relations between extended co-existing objects and causal processes among them not as evidence, but as the determining feature of space and time. In this manner both space and time became tangible and visible to human beings.

What then can physics tell us about the nature of time? The answer is presumably that at this point it can say very little. It seems, first and foremost, to be a metaphysical question whether time is an abstract or concrete entity. Only if time is a concrete particular does it make sense to think that physics can say something intelligible about time as an empirically accessi- 
ble thing. Nonetheless, I think that we can find some support in physics for the idea that time is an abstract entity. The problem is therefore how to find out whether time is abstract or concrete.

\section{The spatio-temporal criterion}

When speaking of abstract objects we usually take them to be objects which purportedly do not exist in space and time. A concrete object is then understood as an object which does exist in space and time. Indeed by this criterion, it is obvious that neither space nor time can be a concrete object, because Space and Time cannot exist in space and time. If this assertion needs an argument, it might be this: First, we must distinguish between time instants and events. An event as a concrete entity is in time. This is why events can be timed, that is, being signed to happen at certain time instants. As two separate entities events and time instants can be related one to one, and therefore timing an event consists in relating an event with a time instant. Normally, we time an event by specifying the moment when the event begins and when it ends. Here clocks and watches help us to mark the right number of moments. Their indications, as events, always represent time instants without problems because such instants are available prior to the timing. For, to say that an event began at a certain time means that it did not exist at a time earlier than this moment.

Analogously, let us assume that Time is a concrete object, and therefore that Time is in time. We must then be able to time Time. But how should this operation be possible? It would require that we could assign moments to the beginning of Time and the end of Time. Again, saying that Time has a beginning at a certain moment means that Time did not exist at an earlier time, and similarly, mutatis mutandis, by saying that Time has an end. Obviously, it means that we need some other time in virtue of which Time can be timed, and this other time must be separable from Time to establish a pairwise relation between them. But we are now moving towards an infinite regress. Such a result is most unwelcome, and to avoid it, we are forced to say that Time cannot exist in time. Thus, time is not a concrete, but an abstract object.

This conclusion might be attacked head on. In a private communication Robin Le Poidevin has launched the following argument in favour of time as a concrete entity: 
(1) Anything whose parts are concrete is itself concrete.

(2) Something is concrete iff it is in time.

(3) Instants are in time, by virtue of standing in temporal relations to other times/events.

Therefore $(2,3)$ :

(4) Instants are concrete.

(5) Instants are parts of time.

Therefore $(1,4,5)$ : time is concrete.

The argument is valid as it stands. We must therefore look at the premises to see which one we may be able to challenge. Both (1) and (2) are acceptable, (2) is even one of paradigmatic criteria of concreteness. This leads us to (3). I think there are ways to counter it.

First, one can accept Jonathan Lowe's suggestion that "perhaps it is a mistake to reify moments of time-to treat them as real entities, to be included in our ontology along with events and persisting objects". (Lowe, 2002: p.315) Second, perhaps in support of this suggestion, one could argue that instants of time are not in time. Events are in time, but not moments themselves. It makes sense to say about some parts that they are in the entity of which they are parts, but there are other parts where it does not make much sense to talk about them as being 'in' or 'contained in' what they are part of. My heart is a part of my body and also placed in the body, whereas my arm is not 'in' my body though it is a part of it. I believe that instants belong to the second category. It is also the case that my arm stands in relation to other bodily parts; say, my left arm stands in relation to my right arm without any of them being 'in' my body. Similarly, everybody agrees that the Battle of Berlin is a part of World War II, although few would be tempted to say that the Battle of Berlin is 'in' World War II and literally mean what is a spatial metaphor. Nevertheless, the Battle of Berlin stands in relations to other battles which happened during World War II.

The lack of congruence between being a part and being 'in' holds for concrete entities like events and persisting objects. The same is true for abstract objects. Number 2 is a part of the numbers between 1 and 10, it stands in relations to all of these numbers, and we can say that number 2 belongs to this series, and that the series consists of numbers from 1 to 10 . But we would not say, other than metaphorically, that number 2 is 'in' this series of numbers. I therefore think that premise (3) in Le Poidevin's argument is not self-promoting in any way and that it requires an independent argument of its own. 


\section{The causal criterion}

There is an alternative, or an additional, criterion of the distinction between the abstract and the concrete. The idea is that concrete objects are those which can participate in causal relations, whereas abstract objects are those which are incapable of entering a causal relationship. Thus a concrete object possesses causal powers or liabilities in contrast to an abstract object that is causally inert. Typically the spatiotemporal and the causal criterion will coincide but there are examples which make it debatable whether this is always the case. We shall therefore see how the latter criterion applies to space and time. Assume that Space and Time are concrete particulars. Then they should be able to causally influence other concrete objects. On the face of it, however, this seems not to be the case. Let us not complicate the matter further and leave out the discussion of space. How, then, can we imagine Time to have a causal influence on things and events? The only way possible, it seems, is if the various time instants somehow causally determine the things and events which occupy them. In principle this should be possible if, say, events and time instants are separate entities which they seem to be. Timing an event does not contribute to the specification of the event. We do not learn anything new about an event to be told that it happened at $t_{1}$ rather than at $t_{2}$. And, likewise, moments exist and can be determinate in relation to other moments independently of any particular events.

So for a time instant to be said to have a causal influence on an event one of two requirements must be fulfilled: Either there must be some properties of this event which it would not have had, unless it happened exactly when it did happen; or it must be the case that the event would have been a different event, if it had happened at a time earlier or later than it actually did. The first condition is excluded by the fact that events and moments are separate entities. Whatever time instant we associate with an event, it does not change the identity or property of this event. Indeed, ascribing a particular date to an event is ascribing a property to that event, but it is a completely contingent and relational property which is not caused by the time instant itself, but by the convention which has been chosen for dating. ${ }^{1}$

Nor can the second requirement be met. This requirement introduces causal overdetermination. Had it not been so, it would exclude material

1 Some may retort that events have the property of being past, present, or future, and this they have because they are affected by time itself. I think, however, this manoeuvre can be blocked, although I shall postpone the discussion until later. 
objects, or other events, from partaking in the causation of events. The causal power of Time should then be able to do the entire work. When it comes to time, however, it seems true to say about any particular event that it could have happened at any specific time without this having any effects on its existence. It is here, I think, we can find support in laws of physics.

The conservation of energy as a fundamental law of physics requires that a system is invariant under translation from one moment to the next. If energy is conserved, it must not make an energy difference in the system at what times the system exists. In other words, Time can have a causal influence on persisting things, only if the translation in time of such things violates the conservation of energy. But we have never observed such a violation, and if we had, the explanation might not be the causality of time. Hence, time also does not stand up to the second criterion of concreteness. We may therefore conclude that time is an abstract entity.

But such a conclusion is still premature. A serious objection lurks in the body of space-time physics. The General Theory of Relativity combines the distribution of matter and energy with the curvature of space-time. In here space-time appears to be treated as a concrete entity which affects matter and energy and is affected by the things in it. There are, I think, several ways of avoiding a claim that the theory affirms the existence of real space-time. One possibility is to "go conventional" with respect to geometry by saying that the actual distribution of matter and energy defines the geometry of the universe in the same way as a force, say in Newton's mechanics, is defined as mass times acceleration. Another way is to argue that the intrinsic geometry of space-time is identified with the structure of the gravitation field but that the latter is concrete whereas the former is not. So what the distribution of mass-energy really determines is the gravitational field, and vice versa. Finally one could argue in line with some philosophers of science that theories should not be considered as literal representations of the world. Rather theories should be taken as a mathematical construction which provides us with a formal language which helps us to construct models by which we explain the behaviour of concrete phenomena (Faye, 2002, ch. 8). It is the second possibility I shall advocate here, though I don't think this excludes the others.

Lawrence Sklar has questioned the causal interpretation of Einstein's field equation: "this idea that the matter distribution "causes the spacetime to have the form it does' must be taken with a grain of salt”. (Sklar, 1974: p.214) Rather he thinks that the equation imposes "a lawlike 'consistency' 
constraint upon the joint features of the world-spacetime structure and mass-energy distribution". (p.74) I read Sklar as saying that Einstein's equation should be regarded as a law of coexistence, and therefore no law of causation is involved, but Robin Le Poidevin read him as if "there is no settling the question of the causal priority between matter distribution and curvature". (p.152) He himself rather believes that "The causal dependence of the geometry of a certain space-time region on a certain distribution of objects in a given region need involve no change in the properties of that region. What we should say, then, is that the distribution of objects in a given region affects curvature of space-time only in the forward light-cone of that region". (p.153) So for his part the conclusion is: “... since local physical geometry is not plausibly thought of as a property of the contents of space-time, in affecting it we are affecting space-time itself". (p.153) But if we think of space-time as an abstract object, it cannot be space-time itself which is affected (nor can space-time cause the distribution of material bodies in it.) What is really affected by the mass-energy distribution is the structure of the gravitational field. Neither can space-time points really be said to coexist with material bodies. What exists together is the gravitational field and material bodies.

\section{The way of abstraction}

So far we have seen that time seems unable to meet the two criteria of concreteness. David Lewis (1986) calls this manoeuvre the way of negation. What we have done is to argue that time lacks some features possessed by paradigmatic concrete things. We have therefore concluded that time is an abstract entity. It would strengthen our interpretation, however, if we also could come up with some positive criteria of abstractness which time is able to meet. The most important one is found in the procedure which Lewis calls the way of abstraction. One form of this procedure has gained much attention in recent years. Already Frege realized that many singular terms that refer to abstract entities are formed by means of functional expressions. Crispin Wright (1983) and Bob Hale (1987) use this idea to develop a theory of abstractness. What Frege had noticed was that we speak of "the number of people", "the direction of a line", and "the shape of an object". Indeed, it is not very uncommon to form singular terms by using functional expressions to refer to concrete objects. For instance, we say "Wittgenstein's father", "the capital of Austria", and "the museums of Vienna". But, 
according to Wright and Hale, the difference is that singular terms denoting abstract objects fit into a formal scheme, sometimes called the abstraction principle:

- $\mathrm{f}(a)=\mathrm{f}(b)$ if and only if $a R b$,

where $R$ is a relation that is reflexive, symmetric and transitive. This relation $R$ is what grounds a sortal $F$, and $R$ does so iff $R$ holds among things such that the truth of statements expressing this fact is sufficient and necessary for the truth of $F$-identity statements. Then, in order for $F$ to be an abstract sortal, it should be possible for $R$ to hold between things which are spatially, but not temporally, separated. (Hale, 1987: p.59) Some further constraints seem plausible to put on $R$. If, on the right side of the equation, $R$ should be considered as the analysans of the meaning of the functional expression on the left side, our understanding of $R$ must semantically, and perhaps even epistemologically, be prior to our understanding of the functional expression.

Let us now try to apply the singular term 'moment' or 'time instant' to the above scheme. Take two distinct events $a$ and $b$. We then get something like:

- The instant of $a=$ the instant of $b$ iff $a$ coexists with $b$.

This shows, I should say, that instant, or moment, fits easily into the abstraction principle. Moreover, the equivalence relation 'to coexist with' seems to be semantically and epistemologically more fundamental than the functional expression.

First, we are able to grasp what it means that two events coexist independently of our understanding of their sharing a common time instant. But to understand the meaning of the statement that two time instants are identical presupposes an understanding of the statement that two events coexist. Terms like 'existence' and 'coexistence' are semantically more basic in the sense that any interpretation of a first-order logical language presupposes a grasp of the timeless existence operator. Also these terms denote something that is epistemologically more basic since we can immediately recognize whether or not two events exist together. Temporal instants are indistinguishable by themselves. Two moments do not posses, qua moment, properties which allow us to identify them independently of persisting clocks and 
events. In fact the expression ' $a$ coexists with $b$ ' means what it does because there is this evidential connection to human experience. Again the upshot seems to be that instants are abstracted entities, and if time consists of instants, then time is a result of such an abstraction.

In return, coexisting events are said to be simultaneous because they share the same time instant. But not all events coexist and therefore do not exist simultaneously. Things undergo change. Because of this we need to be able to talk about how such non-coexisting events relate to one another and how to measure the interval between them. Thus, we create temporal instants by abstraction from a real symmetric relation of 'coexistence'. We then order different time instants with the help of the transitive but asymmetric relation 'is earlier than', or the converse relation 'is later than', and finally we seize the distance between the instants by assigning a metric measure to each of them. We may then introduce expressions like 'past', 'present' and 'future' by saying with respect to every instant that instants, which are earlier than a certain instant, are the past of that instant, and instants, which are simultaneous with this instant, are the present of that instant, and instants, which are later than this instant, are the future of that instant. This is how we arrive at a concept of time as well as tenses.

Since the expression 'is later than', or the converse 'is earlier than', applies to time instants, which are abstracts, it may be taken to stand for an abstract relation. We shall therefore suggest a similar type of analysis of this relation as the one we arrived at before by abstracting from a real asymmetric relation of succession.

- $\mathrm{f}(b)>\mathrm{f}(a)$ iff $\mathrm{f}(b) \neq \mathrm{f}(a) \& b R a$

Now $R$ is not an equivalence relation but an asymmetric, reflexive, and transitive relation. The formula then reads:

- The instant of $b>$ the instant of $a$ iff $b$ does not coexist with $a$, and $b$ succeeds $a$.

This suggestion seems to run into a problem. Events are nothing but changes of persisting things and these succeed one another. Such a relation of succession is real as long as the changes are caused by other changes. Changes come about because the properties of things are able to determine other things to have certain properties. But $a$ need not actually cause $b$, nor be 
even able to do so. This is not a problem, I believe, because if $b$ succeeds $a$, there is always another event $c$, which coexists with $a$, and which causes $b$. If one thinks this still creates a problem, it is possible to introduce talk about the collections of coexisting events instead of particular events. Either way, the understanding of the expression 'succeed' is semantically prior to the expression 'is later than', the truth of the sentence ' $b$ is later than $a$ ' rests entirely on the truth of ' $b$ succeeds $a$ ', and epistemologically we recognize the truth of the former in virtue of the latter as long as we have direct empirical access to $a$ and $b$.

What kind of abstract object is time then? The word 'time' is an abstract singular term like 'wisdom', 'courage', and 'mankind', it is a name, but what kind of object does it refer to? There are several candidates. It could be a universal. But Kant's objection still holds. The relationship between time and its various moments is not of the same kind as between a universal and the various items that exemplify or instantiate it. Another possibility is that time is a set, namely a set of events, or if you like the set of all things having a beginning and possibly an ending. Events and things exist in time, and therefore they are members of such a set. This set is also an ordered set. So 'time' is the term that refers to the collection of all happenings which can be ordered in terms of 'later than' or 'earlier than'.

\section{Some consequences}

The advantages of the present proposal are at least threefold. It explains why the introduction in the Special Theory of Relativity of an operational definition of simultaneity works. If our concept of time instants is abstracted from the concept of coexisting events, it depends on the meaning of coexistence which events can be regarded as simultaneous and which not, but again the meaning of coexistence partly depends on the observed existence of the events. When two distinct events are in close vicinity of each other, we can visually establish whether or not they exist together. But this is not possible when they are separated by a long distance. We therefore need a definition of what we mean by saying that spatially separated events coexist, and such a definition should refer to empirically accessible elements by which we can determine whether or not the existence of two events fall under the definition. Thus the introduction of clocks and light rays in the Special Theory of Relativity set up such a proper definition and provide us with the necessary operational criterion of 'coexistence'. There simply is no abso- 
lute present to which we may appeal in order to establish coexistence since the notion is based on an abstraction; a wrong abstraction nonetheless.

The second advantage is that it is possible to shed new light on McTaggart's argument for the non-existence of time. He assumed that we could have no time instants without events. Even though he maintained that events form the content of time instants there is no empty time; i.e., time instants which are lacking their content of events. Thus being past, present, and future, according to McTaggart, consists of a set of relations between events which are constantly changing. So when events change, time instants change. In this way McTaggart presupposed time to be a concrete entity because time instants continuously change their properties from being more and less future to being present, and again from being present to being less and more past. But a specific time instant cannot change its properties in relation to another specific time instant. Therefore he came up with an inconsistency or a regress. But if we accept that time instants do not change properties or relations, but that only concrete objects are able to undergo change, there is no longer an inconsistency involved. And by assuming that time instants are abstractions, we can explain why time instants cannot change their relations to other time instants. As abstract relations between abstract entities they are timelessly permanent. So McTaggart's argument simply showed that time cannot be a concrete entity and therefore is not a part of (physical) reality.

This leads us to the third issue in the debate about becoming or the passage of time. In my opinion the proposal helps us to vindicate the detenser's talk about the tenseless existence of events. It is because every event is a member of an ordered set of events that an event can be said to exist tenselessly. Time is a set of events ordered by the relation 'earlier than' or 'later than', but an ordering of time instants is possible because some facts hold about the related items. Such facts are usually taken to concern the internal properties of the events involved. It cannot be past, present, or future, since, as I argued, these are 2-rank abstract relations based on 1-rank abstract relations such as earlier than or later than. But neither is the relation later than an internal relation, because existence simpliciter, and therefore coexistent, is not a property of the event. As a consequence, the relation between time instants cannot change in accordance with the property of its relata. The facts are rather that the items, which do not coexist, permanently do not coexist, thus permanently exist at different times. The ordering relation 'is later than' is a permanent relation which holds between time instants, i.e., 
between different subsets of coexisting events, and this requires that events exist tenselessly whenever they occur. We shall define tenseless occurrence in this way: an event exists tenselessly at a time $t$ if, and only if, it exists at $t$ with respect to any earlier time $t^{*}$ and with respect to any later time $t^{* *}$. I think that most proponents of the tenseless view could subscribe to this ontological definition (or some equivalent semantic formulation.) Now, saying that an event occurs tenselessly at $t$ entails, of course, that the event neither occurs tenselessly at $t^{*}$ earlier than $t$, or occurs tenselessly at $t^{* *}$ later than $t$. The event only exists at $t$ in virtue of coexisting with other events which all belong to the same subset that grounds the time instants $t$. The implication is that events existing tenselessly at different times do not in any sense coexist because they belong to different subsets of coexisting events.

In a recent paper Jonathan Lowe (2005) has suggested that time is not a dimension of reality (in contrast to spatial dimensions.) I very much agree. I think that neither he nor I coexist with Julius Caesar, and I also think that the four-dimensional representation of the world is an abstraction. But my argument for saying this is different. Since I take time to be an abstract entity formed to talk about persisting things and changes, I also think there has to be some real relation apart from the relation of coexistence. Lowe maintains that "if entities are differently located along some real dimension, they must stand in a certain real (external) relations to one another, which they can do only if they are in some sense co-existent. For a real relation can obtain only between entities all of which exist together". (p.14) A little later he writes "inasmuch as four-dimensionalism holds that persisting objects are composed of entities that exist only at different moments of time, it certainly seems committed to regarding time as a dimension in which reality is extended". I accept that. But he then continues: "This is because any principle of composition necessarily invokes a relation between the composing entities and thereby requires those entities to co-exist as the relata of that relation". (p.17) I would deny this and claim instead that it only requires that these entities exist tenselessly.

The point is that we need a real relation other than the relation of coexistence which enables us to talk about change and order of events which do not exist together. This is the relation of succession. The relata of that relation do not coexist. They belong to two different sets of coexisting events. So if we accept that any principle of composition requires the existence of the relata, it only follows, I think, that the relata must exist tenselessly with respect to each other. Following the way of abstraction we have that the 
statement ' $a$ exists tenselessly at $t$ ' is true if and only if $a$ coexists tenselessly with another event $b$; and again, the statement ' $a$ coexists tenselessly with $b$ ' is true if and only if $a$ and $b$ coexist with respect to each other but also coexist together with respect to all those events which they do not coexist with. Thereby we can explain why it is tenselessly true to say that 'Caesar's life is/ was earlier than mine', 'Caesar lived before me', and 'I will die sometimes after I have written these lines'.

So apart from the relation of coexistence we have to introduce the relation of succession in order to make it possible to ground time-talk to reality. And we need a real relation of succession if we do not want to make different subsets of coexisting events ontologically isolated from one another. But the relata of this relation cannot themselves coexist. Thus they have to exist tenselessly. It is in this minimal sense that we have objective becoming.

\section{Conclusion}

I have presented some arguments according to which time is not a concrete entity of the world. By saying that time is an abstract entity I do not claim that times exist quite independently of what they are abstracted from or independently of human beings doing the abstraction. I do not feel committed to a Platonic world of abstract objects. Nor do I think that abstracts are reducible to concretes. We need abstractions because they make it possible for us to characterize a collection of concrete entities or properties instead of particular things or properties. Stepping down for the first time on the surface of the Moon, Neil Armstrong said the now famous words: "That's one small step for a man, one giant leap for mankind". As an abstract object mankind has properties which no single person can ever have. It is not different when it comes to time. Time-talk allows us to organize and structure events and changes which we cannot access by our immediate senses.

Acknowledgement: I wish to thank Robin Le Poidevin for his helpful comments on my talk at Kirchberg. 


\section{Literature}

Earman, John 1989 World Enough and Space-Time. Boston: MIT Press.

Faye, Jan 2002 Rethinking Science, Aldershot: Ashgate Publisher.

Hale, Bob 1987 Abstract Objects. Oxford: Basil Blackwell.

Kant, Immanuel 1933 Critique of the Pure Reason. Trans. Norman Kemp Smith. London: Macmillian.

Le Poidevin, Robin 1992 'On the Acausality of Time, Space and SpaceTime', Analysis 52, 146-54.

Lewis, David 1986 On the Plurality of Worlds. Oxford: Basil Blackwell.

Lowe, Jonathan 2002 A Survey of Metaphysics. Oxford: Oxford University Press.

Lowe, Jonathan 2005 'How Real Is Substantial Change?' (forthcoming in The Monist)

Sklar, Lawrence 1974 Space, Time and Spacetime. Berkeley: University of California Press.

Wright, Crispin 1983 Frege's Conception of Numbers as Objects, Aberdeen: Aberdeen University Press. 